\title{
Vector SUSY models with Carroll or Galilei invariance
}

\author{
Andrea Barducci* and Roberto Casalbuoni ${ }^{\dagger}$ \\ Department of Physics, University of Florence and INFN, Via G. Sansone 1, \\ 50019 Sesto Fiorentino (FI), Italy \\ Joaquim Gomis \\ Departament de Física Quàntica i Astrofísica and Institut de Ciències del Cosmos (ICCUB), \\ Universitat de Barcelona Martí i Franquès, ES-08028 Barcelona, Spain
}

(Received 21 December 2018; published 22 February 2019)

\begin{abstract}
The general method introduced in a previous paper to build up a class of models invariant under generalization of Carroll and Galilei algebra is extended to systems including a set of Grassmann variables describing the spin degree of freedom. The models described here are based on a relativistic supersymmetric algebra with vector and scalar generators (VSUSY). Therefore, in order to obtain dynamical systems consistent with Carroll or Galilei, we will study the contractions of the anticommuting generators compatible with the Poincaré contractions.
\end{abstract}

DOI: 10.1103/PhysRevD.99.045016

\section{INTRODUCTION}

In a previous paper [1] we have introduced a general strategy to build up a class of models invariant under generalizations of Carroll and Galilei algebra with zero central charge [2-10] A bonus of this approach is that it allows a description in configuration space, whereas most of the models invariant under Carroll or Galilei group present in the literature are described by an action in phase space. Furthermore, although the construction of these nonrelativistic actions starts from a relativistic formulation, the method in [1] does not require any limiting procedure and redefinition of the parameters.

The aim of this work is to apply this method to systems including a set of Grassmann variables describing the spin degrees of freedom, exhibiting a Carroll or a Galilei symmetry. The system we have in mind is one with a SUSY symmetry described by vectorlike and scalar anticommuting generators (VSUSY). This algebra, which is a relativistic one, was introduced in [11], in order to get a "pseudoclassical" description of the Dirac equation. See also [12-14], where these pseudoclassical models have the same set of Grassmann variables and lead to the Dirac equation without rigid supersymmetry.

\footnotetext{
*barducci@fi.infn.it

†casalbuoni@fi.infn.it

joaquim.gomis@ub.edu
}

Published by the American Physical Society under the terms of the Creative Commons Attribution 4.0 International license. Further distribution of this work must maintain attribution to the author(s) and the published article's title, journal citation, and DOI. Funded by SCOAP ${ }^{3}$.
VSUSY has been introduced in Ref. [1] in connection with the idea of describing the spin degree of freedom in terms of Grassmann variables. Correspondingly, VSUSY is a nontrivial extension of the Poincaré group via the introduction of anticommuting generators. By its very nature VSUSY is applicable to particle-like objects. The extension to field theory, considering the spin statistics connection, is non trivial. The connection among ordinary QFT and TQFT is based on the use of twisting where spinorial charges are changed to vectors, scalars, and selfdual tensors charges at the level of the superparticle with $N=2$, see [15]. Also Costello constructed twisted supergravity [16]. The general algebraic properties of VSUSY have been studied in Ref. [17]. In particular, the Casimir operators have been constructed and the structure of the multiplets has been investigated. Furthermore, it has been shown that this algebra can be obtained from a contraction of $\operatorname{OSp}(3,2 \mid 2)$.

Of course, the models we would like to construct here should exhibit Carroll or Galilei supersymmetry counterpart. To this end, we would need to construct specific contractions of VSUSY algebra. Before doing that, we would need to review the general strategy of [1].

The method consists in starting from a space-time in $D+1$ dimensions and partitioning it in two parts, the first Minkowskian and the second euclidean. Then a Carroll or Galilei invariant model can be obtained by introducing a Minkowski invariant action, or Euclidean invariant action respectively, in one of the partitions of the space-time (the Minkowskian for Carroll or the euclidean for Galilei) and, in the complementary partition, a system of lagrange multipliers transforming in an appropriate way under the 
Euclidean or the Lorentz group such that to confine the system to a region of the space-time. This system is such to compensate the variations induced by the Carroll or Galilei boosts of the action in the appropriate subspace. A simple way to get this result, is to start with an action Poincaré invariant in the total space, say $S$, and defining the action in the Minkowski or euclidean subspace as

$$
(S)_{\text {Carroll }}=\left.(S)\right|_{x^{a} \equiv 0},
$$

or

$$
(S)_{\text {Galilei }}=\left.(S)\right|_{x^{\alpha} \equiv 0},
$$

where the $x^{a}$ 's and $x^{\alpha}$ 's are the coordinates of the Euclidean subspace where the system is confined respectively for the Carroll and Galilei case.

It is important to notice that this procedure allows us to obtain the action, for dynamical systems invariant under Carroll or Galilei group without central charges, directly from actions in configurations space invariant under Poincaré without use of a limiting procedure. As we have said one needs to introduce a set of Lagrange multipliers in one of the two parts of the Lagrangian in which we divide the space-time. These multipliers have not a clear interpretation in configuration space. However, in general, these Lagrange multipliers have a natural interpretation as momenta in the phase space canonical action.

The paper is organized as follows: in Sec. II we review the $k$-contractions [18-22] [7-10] used in Ref. [1]. In Secs. III A-III B, we construct the $k$-contractions of VSUSY algebra for the Carroll type and the Galilei type, both in the case of the abstract algebra and in the case of the realization of the algebra in the configuration space. In Sec. IV, following the procedure illustrated in [1] we construct the action and discuss the case for a VSUSY Carroll particle. In particular we examine its $\kappa$ invariance and its quantization respectively in Secs. IVA and IV B. The same is done for a VSUSY Galilei particle in Secs. V, VA, and V B In Sec. VI we draw our conclusions. In the Appendix we show how the actions, for the VSUSY Carroll and Galilei particle, can be derived by performing the standard Carroll and nonrelativistic limiting procedures in the Poincaré invariant phase space action.

\section{POINCARÉ CONTRACTIONS}

In order to make explicit the partition of the space-time in $D+1$ dimensions we will introduce the following set of coordinates

$$
\begin{aligned}
& \mu, \nu=0,1, \ldots, D, \quad \eta_{\mu \nu}=(-;+, \cdots,+), \\
& \alpha, \beta=0,1, \ldots, k-1, \quad \eta_{\alpha \beta}=(-;+, \cdots,+), \\
& a, b=k, \ldots, D, \quad \eta_{a b}=(+,+, \cdots,+),
\end{aligned}
$$

The Poincaré algebra in the total space is given by

$$
\begin{aligned}
{\left[M_{\mu \nu}, M_{\rho \sigma}\right] } & =-i\left(\eta_{\mu \rho} M_{\nu \sigma}+\eta_{\nu \sigma} M_{\mu \rho}-\eta_{\mu \sigma} M_{\nu \rho}-\eta_{\nu \rho} M_{\mu \sigma}\right) \\
{\left[M_{\mu \nu}, P_{\rho}\right] } & =-i\left(\eta_{\mu \rho} P_{\nu}-\eta_{\nu \rho} P_{\mu}\right) \\
{\left[P_{\mu}, P_{\nu}\right] } & =0
\end{aligned}
$$

Then, consider the following two subgroups of $I S O(1, D)$ : the Poincare subgroup in $k$ dimensions, $I S O(1, k-1)$ and the Euclidean group of roto-translations in $D+1-k$ dimensions, generated respectively by

$$
\begin{array}{r}
I S O(1, k-1): M_{\alpha \beta}, \quad P_{\alpha}, \quad \alpha, \beta=0,1, \ldots, k-1, \\
I S O(D+1-k): M_{a b}, \quad P_{a}, \quad a, b=k, \ldots, D .
\end{array}
$$

In these notations the generators of $I S O(1, D)$ are

$I S O(1, D): M_{\alpha \beta}, \quad M_{a b}, \quad P_{\alpha}, \quad P_{a}, \quad M_{\alpha b} \equiv B_{\alpha b}$.

Note that the generators $B_{\alpha b}$ connect the two subalgebras.

In [1] we have considered two types of contractions, both at the level of the Poincaré algebra and at the level of the invariant vector fields. These contractions generalize the Carroll [2,5-7] and the Galilei algebras [23] [18-22] [8-10].

At the Lie algebra level the contractions are made on the momenta and on the boosts; in the Carroll case:

$$
\tilde{P}_{\alpha}=\frac{1}{\omega} P_{\alpha}, \quad \tilde{B}_{\alpha a}=\frac{1}{\omega} B_{\alpha a}
$$

and then taking the limit $\omega \rightarrow \infty$. In the Galilei case the contractions are given by

$$
\tilde{P}_{a}=\frac{1}{\omega} P_{a}, \quad \tilde{B}_{\alpha a}=\frac{1}{\omega} B_{\alpha a} .
$$

The commutation relations resulting from the contraction process are

Carroll-type

$$
\left(\begin{array}{c|ccc} 
& B_{\beta b} & P_{\beta} & P_{b} \\
\hline B_{\alpha a} & 0 & 0 & i \eta_{a b} P_{\alpha} \\
P_{\alpha} & 0 & 0 & 0 \\
P_{a} & -i \eta_{a b} P_{\beta} & 0 & 0
\end{array}\right),
$$

Galilei-type

$$
\left(\begin{array}{c|ccc} 
& B_{\beta b} & P_{\beta} & P_{b} \\
\hline B_{\alpha a} & 0 & -i \eta_{\alpha \beta} P_{a} & \\
P_{\alpha} & i \eta_{\alpha \beta} P_{a} & 0 & 0 \\
P_{a} & 0 & 0 & 0
\end{array}\right) .
$$




\section{VSUSY CONTRACTIONS}

Let us now consider the VSUSY algebra [11,24] which is defined in terms of the generators $G_{\mu}$ and $G_{5}$ and of their anticommutation relations

$$
\begin{gathered}
{\left[G_{\mu}, G_{\nu}\right]_{+}=\eta_{\mu \nu} Z, \quad\left[G_{\mu}, G_{5}\right]_{+}=-P_{\mu},} \\
{\left[G_{5}, G_{5}\right]_{+}=Z_{5}}
\end{gathered}
$$

By using a decomposition analogous to the one used in the Poincaré case

$G_{\mu}=\left(G_{\alpha}, G_{a}\right), \quad \alpha=0,1, \ldots, k-1, \quad a=k, \ldots, D$

The anticommutation relations become

$$
\begin{gathered}
{\left[G_{\alpha}, G_{\beta}\right]_{+}=\eta_{\alpha \beta} Z, \quad\left[G_{a}, G_{b}\right]_{+}=\eta_{a b} Z,} \\
{\left[G_{5}, G_{5}\right]_{+}=Z_{5}} \\
{\left[G_{\alpha}, G_{a}\right]_{+}=0, \quad\left[G_{\alpha}, G_{5}\right]_{+}=-P_{\alpha},} \\
{\left[G_{a}, G_{5}\right]_{+}=-P_{a}}
\end{gathered}
$$

Both $G_{\alpha}$ and $G_{a}$ behave as vectors under the two groups generated by $M_{\alpha \beta}$ and $M_{a b}$. Therefore the corresponding commutation relations are invariant under any rescaling of $G_{\alpha}$ and $G_{a}$. This is not the same for the Lorentz boosts with commutation relations

$$
\left[B_{\alpha a}, G_{\beta}\right]=+i \eta_{\alpha \beta} G_{a}, \quad\left[B_{\alpha a}, G_{b}\right]=-i \eta_{a b} G_{\alpha}
$$

\section{A. $k$-contractions of Carroll type}

In the Poincaré case we have defined the contraction by a rescaling of part of the momenta and for the boosts, according to the Eq. (8) for the Carroll case and Eq. (9) for the Galilei one, leaving unchanged all the other generators. By doing so we have not changed the two subalgebras $\operatorname{ISO}(1, k-1)$ and $\operatorname{ISO}(D+1-k)$. In the case of VSUSY we would like to make use of an analogous strategy. In particular, for the Carroll case, we would like to maintain the VSUSY invariance in the Minkowski sector. Therefore we will study $k$-contractions of Carroll type, preserving the main anticommutation relation in this sector:

$$
\left[G_{\alpha}, G_{5}\right]_{+}=-P_{\alpha} .
$$

To this end we will define the contracted generators as

$$
\tilde{G}_{\alpha}=\frac{1}{\omega^{r}} G_{\alpha}, \quad \tilde{G}_{a}=\frac{1}{\omega^{t}} G_{a}, \quad \tilde{G}_{5}=\frac{1}{\omega^{1-r}} G_{5} .
$$

With this choice we have

$$
\begin{gathered}
{\left[\tilde{G}_{\alpha}, \tilde{G}_{\beta}\right]_{+}=\eta_{\alpha \beta} \frac{Z}{\omega^{2 r}}, \quad\left[\tilde{G}_{a}, \tilde{G}_{b}\right]_{+}=\eta_{a b} \frac{Z}{\omega^{2 t}},} \\
{\left[\tilde{G}_{5}, \tilde{G}_{5}\right]_{+}=\frac{Z_{5}}{\omega^{2-2 r}}}
\end{gathered}
$$

$$
\left[\tilde{G}_{\alpha}, \tilde{G}_{5}\right]_{+}=-\tilde{P}_{\alpha}, \quad\left[\tilde{G}_{a}, \tilde{G}_{5}\right]_{+}=-\frac{1}{\omega^{1-r+t}} \tilde{P}_{a}
$$

$\left[\tilde{B}_{\alpha a}, \tilde{G}_{\beta}\right]=-\frac{i}{\omega^{1+r-t}} \eta_{\alpha \beta} \tilde{G}_{a}, \quad\left[\tilde{B}_{\alpha a}, \tilde{G}_{b}\right]=+\frac{i}{\omega^{1-r+t}} \eta_{a b} \tilde{G}_{\alpha}$

where, for the moment being we have not done any choice regarding the possible contractions of the central charges. Asking to maintain the complete VSUSY algebra in the Minkowski subspace, we should scale both $Z$ and $Z_{5}$

$$
\tilde{Z}=\frac{Z}{\omega^{2 r}}, \quad \tilde{Z}_{5}=\frac{Z_{5}}{\omega^{2-2 r}} .
$$

Then, we have

$$
\begin{gathered}
{\left[\tilde{G}_{\alpha}, \tilde{G}_{\beta}\right]_{+}=\eta_{\alpha \beta} \tilde{Z}, \quad\left[\tilde{G}_{a}, \tilde{G}_{b}\right]_{+}=\eta_{a b} \frac{\tilde{Z}}{\omega^{2(t-r)}},} \\
{\left[\tilde{G}_{5}, \tilde{G}_{5}\right]_{+}=\tilde{Z}_{5}} \\
{\left[\tilde{G}_{\alpha}, \tilde{G}_{5}\right]_{+}=-\tilde{P}_{\alpha}, \quad\left[\tilde{G}_{a}, \tilde{G}_{5}\right]_{+}=-\frac{1}{\omega^{1-r+t}} \tilde{P}_{a}} \\
{\left[\tilde{B}_{\alpha a}, \tilde{G}_{\beta}\right]=-\frac{i}{\omega^{1+r-t}} \eta_{\alpha \beta} \tilde{G}_{a}, \quad[24} \\
{\left[\tilde{B}_{\alpha a}, \tilde{G}_{b}\right]=+\frac{i}{\omega^{1-r+t}} \eta_{a b} \tilde{G}_{\alpha} .}
\end{gathered}
$$

Now, all these relations depend only on the difference $r-t$. To have a finite result, it is enough to require $t-r \geq 0$ [as it follows from (23)] and, from (25), $1+r-t \geq 0$. There are only two possibilities to verify these conditions:

(1) $t-r=0$, the relations become

$$
\begin{gathered}
{\left[\tilde{G}_{\alpha}, \tilde{G}_{\beta}\right]_{+}=\eta_{\alpha \beta} \tilde{Z}, \quad\left[\tilde{G}_{a}, \tilde{G}_{b}\right]_{+}=\eta_{a b} \tilde{Z}, \quad\left[\tilde{G}_{5}, \tilde{G}_{5}\right]_{+}=\tilde{Z}_{5}} \\
{\left[\tilde{G}_{\alpha}, \tilde{G}_{5}\right]_{+}=-\tilde{P}_{\alpha}, \quad\left[\tilde{G}_{a}, \tilde{G}_{5}\right]_{+}=0} \\
{\left[\tilde{B}_{\alpha a}, \tilde{G}_{\beta}\right]=0, \quad\left[\tilde{B}_{\alpha a}, \tilde{G}_{b}\right]=0}
\end{gathered}
$$

Notice that the $G_{a}$ 's form a Clifford algebra (after renormalization of the generators) which commute or anticommute with all other generators of the previous list. The algebra is VSUSY $\otimes$ Clifford, with VSUSY in $k$-dimensions and the Clifford in $D+1-k$ dimensions. 
2) $t-r=1$, we get

$$
\begin{gathered}
{\left[\tilde{G}_{\alpha}, \tilde{G}_{\beta}\right]_{+}=\eta_{\alpha \beta} \tilde{Z}, \quad\left[\tilde{G}_{a}, \tilde{G}_{b}\right]_{+}=0, \quad\left[\tilde{G}_{5}, \tilde{G}_{5}\right]_{+}=\tilde{Z}_{5}} \\
{\left[\tilde{G}_{\alpha}, \tilde{G}_{5}\right]_{+}=-\tilde{P}_{\alpha}, \quad\left[\tilde{G}_{a}, \tilde{G}_{5}\right]_{+}=0} \\
{\left[\tilde{B}_{\alpha a}, \tilde{G}_{\beta}\right]=-i \eta_{\alpha \beta} \tilde{G}_{a}, \quad\left[\tilde{B}_{\alpha a}, \tilde{G}_{b}\right]=0}
\end{gathered}
$$

Here the $\tilde{G}_{a}$ 's span a Clifford algebra with zero central charge, or a Grasmmann algebra. In the case $r=t$, corresponding to the model discussed in the following, the boosts connect only the space-time variables among the two sectors.

\section{B. $\boldsymbol{k}$-contractions of Carroll type in configuration space}

The scaling of the algebra of the generators, implies the following scaling in the configuration space realization

$$
\begin{aligned}
& \tilde{x}^{\alpha}=\omega x^{\alpha}, \quad \tilde{x}^{a}=x^{a}, \quad \tilde{\xi}^{\alpha}=\omega^{r} \xi^{\alpha}, \quad \tilde{\xi}^{a}=\omega^{t} \xi^{a}, \\
& \tilde{\xi}^{5}=\omega^{1-r} \xi^{5}, \quad \tilde{c}=\omega^{2 r} c, \quad \tilde{c}_{5}=\omega^{2-2 r} c_{5}
\end{aligned}
$$

We will make use of the vector field realization of the VSUSY algebra, as given [24]:

$G_{\mu}=-i\left(\frac{\partial}{\partial \xi^{\mu}}+i \xi^{5} \frac{\partial}{\partial x^{\mu}}-\frac{i}{2} \xi_{\mu} \frac{\partial}{\partial c}\right), \quad G_{5}=-i \frac{\partial}{\partial \xi^{5}}-\frac{1}{2} \xi^{5} \frac{\partial}{\partial c_{5}}$

with the generators of the Poincare group in the $D+1$ dimensional space:

$$
\begin{aligned}
M_{\mu \nu} & =-i\left(x_{\mu} \frac{\partial}{\partial x^{\nu}}-x_{\nu} \frac{\partial}{\partial x^{\mu}}\right)-i\left(\xi_{\mu} \frac{\partial}{\partial \xi^{\nu}}-\xi_{\nu} \frac{\partial}{\partial \xi^{\mu}}\right), \\
P_{\mu} & =-i \frac{\partial}{\partial x^{\mu}} .
\end{aligned}
$$

Notice that the derivatives with respect to the Grassmann variables are left-handed derivatives.

Performing the scaling on the vector fields according to Eqs. (18), (8), and (66) we get for the two cases

(1) $t-r=0$,

$$
\begin{gathered}
\tilde{G}_{\alpha}=-i\left(\frac{\partial}{\partial \tilde{\xi}^{\alpha}}+i \tilde{\xi}^{5} \frac{\partial}{\partial \tilde{x}^{\alpha}}-\frac{i}{2} \tilde{\xi}_{\alpha} \frac{\partial}{\partial \tilde{c}}\right) \\
\tilde{G}_{a}=-i\left(\frac{\partial}{\partial \tilde{\xi}^{a}}-\frac{i}{2} \tilde{\xi}_{a} \frac{\partial}{\partial \tilde{c}}\right) \\
\tilde{G}_{5}=-i \frac{\partial}{\partial \tilde{\xi}^{5}}-\frac{1}{2} \tilde{\xi}^{5} \frac{\partial}{\partial \tilde{c}_{5}} \\
\tilde{B}_{\alpha a}=i \tilde{x}_{a} \frac{\partial}{\partial \tilde{x}^{\alpha}}
\end{gathered}
$$

Implying the following variations for the coordinates (omitting the tilde)

$$
\begin{gathered}
i \epsilon^{\alpha} G_{\alpha}: \delta \xi^{\alpha}=\epsilon^{\alpha}, \quad \delta x^{\alpha}=i \epsilon^{\alpha} \xi^{5}, \quad \delta c=-\frac{i}{2} \epsilon^{\alpha} \xi_{\alpha} \\
i \epsilon^{a} G_{a}: \delta \xi^{a}=\epsilon^{a}, \quad \delta x^{a}=0, \quad \delta c=-\frac{i}{2} \epsilon^{a} \xi_{a} \\
i \epsilon^{5} G_{5}: \delta \xi^{5}=\epsilon^{5}, \quad \delta c_{5}=-\frac{i}{2} \epsilon^{5} \xi^{5} \\
i v^{\alpha a} B_{\alpha a}: \delta x^{\alpha}=-v^{\alpha a} x_{a}
\end{gathered}
$$

(2) $t-r=1$, we get

$$
\begin{gathered}
\tilde{G}_{\alpha}=-i\left(\frac{\partial}{\partial \tilde{\xi}^{\alpha}}+i \tilde{\xi}^{5} \frac{\partial}{\partial \tilde{x}^{\alpha}}-\frac{i}{2} \tilde{\xi}_{\alpha} \frac{\partial}{\partial \tilde{c}}\right) \\
\tilde{G}_{a}=-i \frac{\partial}{\partial \tilde{\xi}^{a}} \\
\tilde{G}_{5}=-i \frac{\partial}{\partial \tilde{\xi}^{5}}-\frac{1}{2} \tilde{\xi}^{5} \frac{\partial}{\partial \tilde{c}_{5}} \\
\tilde{B}_{\alpha a}=i \tilde{x}_{a} \frac{\partial}{\partial \tilde{x}^{\alpha}}-i \tilde{\xi}_{\alpha} \frac{\partial}{\partial \tilde{\xi}^{a}}
\end{gathered}
$$

Implying the following variations for the coordinates (omitting the tilde)

$$
\begin{gathered}
i \epsilon^{\alpha} G_{\alpha}: \delta \xi^{\alpha}=\epsilon^{\alpha}, \quad \delta x^{\alpha}=i \epsilon^{\alpha} \xi^{5}, \quad \delta c=-\frac{i}{2} \epsilon^{\alpha} \xi_{\alpha} \\
i \epsilon^{a} G_{a}: \delta \xi^{a}=\epsilon^{a}, \quad \delta x^{a}=0, \quad \delta c=0 \\
i \epsilon^{5} G_{5}: \delta \xi^{5}=\epsilon^{5}, \quad \delta c_{5}=-\frac{i}{2} \epsilon^{5} \xi^{5} \\
i v^{\alpha a} B_{\alpha a}: \delta x^{\alpha}=-v^{\alpha a} x_{a}, \quad \delta \xi^{a}=v^{\alpha a} \xi_{\alpha}
\end{gathered}
$$

Note that the difference between the two types of transformations is the action of the boosts. In the actual case, the boosts depend also on the Grassmann variables, $\xi^{\alpha}$ amd $\xi^{a}$, Furtermore the VSUSY algebra decomposes in the direct product of a VSUSY in the Minkowski subspace times a Grassmann algebra in the Euclidean part.

\section{C. $k$-contractions of Galilei type}

In this case the contraction at the level of the Poincare group is defined in Eq. (9). This time we want to maintain the VSUSY invariance in the Euclidean subsector. Therefore we will study the $k$-contractions of Galilei type, preserving the main anticommutation relation: 


$$
\left[G_{a}, G_{5}\right]_{+}=-P_{a} .
$$

To this end we will define the contracted generators as

$\tilde{G}_{\alpha}=\frac{1}{\omega^{r}} G_{\alpha}, \quad \tilde{G}_{a}=\frac{1}{\omega^{t}} G_{a}, \quad \tilde{G}_{5}=\frac{1}{\omega^{1-t}} G_{5}$.

With this choice we have

$$
\begin{gathered}
{\left[\tilde{G}_{\alpha}, \tilde{G}_{\beta}\right]_{+}=\eta_{\alpha \beta} \frac{Z}{\omega^{2 r}}, \quad\left[\tilde{G}_{a}, \tilde{G}_{b}\right]_{+}=\eta_{a b} \frac{Z}{\omega^{2 t}},} \\
{\left[\tilde{G}_{5}, \tilde{G}_{5}\right]_{+}=\frac{Z_{5}}{\omega^{2-2 t}}} \\
{\left[\tilde{G}_{\alpha}, \tilde{G}_{5}\right]_{+}=-\frac{1}{\omega^{1+r-t}} \tilde{P}_{\alpha}, \quad\left[\tilde{G}_{a}, \tilde{G}_{5}\right]_{+}=-\tilde{P}_{a}} \\
{\left[\tilde{B}_{\alpha a}, \tilde{G}_{\beta}\right]=-\frac{i}{\omega^{1+r-t}} \eta_{\alpha \beta} \tilde{G}_{a}, \quad\left[\tilde{B}_{\alpha a}, \tilde{G}_{b}\right]=+\frac{i}{\omega^{1-r+t}} \eta_{a b} \tilde{G}_{\alpha} .}
\end{gathered}
$$

Asking to maintain the complete VSUSY algebra in the Euclidean sector, we need to scale both $Z$ and $Z_{5}$

$$
\tilde{Z}=\frac{Z}{\omega^{2 t}}, \quad \tilde{Z}_{5}=\frac{Z_{5}}{\omega^{2-2 t}}
$$

Then we have

$$
\begin{gathered}
{\left[\tilde{G}_{\alpha}, \tilde{G}_{\beta}\right]_{+}=\eta_{\alpha \beta} \frac{\tilde{Z}}{\omega^{2(r-t)}}, \quad\left[\tilde{G}_{a}, \tilde{G}_{b}\right]_{+}=\eta_{a b} \tilde{Z}} \\
{\left[\tilde{G}_{5}, \tilde{G}_{5}\right]_{+}=\tilde{Z}_{5}} \\
{\left[\tilde{G}_{\alpha}, \tilde{G}_{5}\right]_{+}=-\frac{1}{\omega^{1+r-t}} \tilde{P}_{\alpha}, \quad\left[\tilde{G}_{a}, \tilde{G}_{5}\right]_{+}=-\tilde{P}_{a}} \\
{\left[\tilde{B}_{\alpha a}, \tilde{G}_{\beta}\right]=-\frac{i}{\omega^{1+r-t}} \eta_{\alpha \beta} \tilde{G}_{a}, \quad[58)}
\end{gathered}
$$

Reasoning as in the Carroll case, we have only two possibilities:

(1) $r-t=0$, the relations become

$$
\begin{array}{r}
{\left[\tilde{G}_{\alpha}, \tilde{G}_{\beta}\right]_{+}=\eta_{\alpha \beta} \tilde{Z}, \quad\left[\tilde{G}_{a}, \tilde{G}_{b}\right]_{+}=\eta_{a b} \tilde{Z},} \\
{\left[\tilde{G}_{5}, \tilde{G}_{5}\right]_{+}=\tilde{Z}_{5}} \\
{\left[\tilde{G}_{\alpha}, \tilde{G}_{5}\right]_{+}=0, \quad\left[\tilde{G}_{a}, \tilde{G}_{5}\right]_{+}=-P_{a}} \\
{\left[\tilde{B}_{\alpha a}, \tilde{G}_{\beta}\right]=0, \quad\left[\tilde{B}_{\alpha a}, \tilde{G}_{b}\right]=0 .}
\end{array}
$$

Notice that the $\tilde{G}_{\alpha}$ 's form a Clifford algebra (after renormalization of the generators). The algebra is VSUSY $\otimes$ Clifford, with VSUSY in $D+1-k$-dimensions and the Clifford in $k$ dimensions.
(2) $r-t=1$, we get

$$
\left[\tilde{G}_{\alpha}, \tilde{G}_{\beta}\right]_{+}=0, \quad\left[\tilde{G}_{a}, \tilde{G}_{b}\right]_{+}=\eta_{a b} \tilde{Z}, \quad\left[\tilde{G}_{5}, \tilde{G}_{5}\right]_{+}=\tilde{Z}_{5}
$$

$$
\left[\tilde{G}_{\alpha}, \tilde{G}_{5}\right]_{+}=0, \quad\left[\tilde{G}_{a}, \tilde{G}_{5}\right]_{+}=-P_{a}
$$

$$
\left[\tilde{B}_{\alpha a}, \tilde{G}_{\beta}\right]=0, \quad\left[\tilde{B}_{\alpha a}, \tilde{G}_{b}\right]=i \eta_{a b} \tilde{G}_{\alpha}
$$

Here the $\tilde{G}_{\alpha}$ 's span a Clifford algebra with zero central charge, or a Grasmmann algebra. Then we can make considerations analogous to the ones made in the Carroll case. That is in the first case (corresponding to the model discussed in the following), the boosts connect only the space-time variables among the two sectors.

\section{D. $k$-contractions of Galilei type in configuration space}

The scaling of the algebra of the generators, implies the following scaling in the configuration space

$$
\begin{array}{lll}
\tilde{x}^{a}=\omega x^{a}, & \tilde{\xi}^{\alpha}=\omega^{r} \xi^{\alpha}, & \tilde{\xi}^{a}=\omega^{t} \xi^{a}, \\
\tilde{\xi}^{5}=\omega^{1-t} \xi^{5}, & \tilde{c}=\omega^{2 t} c, & \tilde{c}_{5}=\omega^{2-2 t} c_{5}
\end{array}
$$

Recalling the expressions (33) and (34) we perform the scaling on the expression of the generators according to Eqs. (52), (56), and (9). The result is

(1) $r-t=0$,

$$
\begin{gathered}
\tilde{G}_{\alpha}=-i\left(\frac{\partial}{\partial \tilde{\xi}^{\alpha}}-\frac{i}{2} \tilde{\xi}_{\alpha} \frac{\partial}{\partial \tilde{c}}\right) \\
\tilde{G}_{a}=-i\left(\frac{\partial}{\partial \tilde{\xi}^{a}}+i \tilde{\xi}^{5} \frac{\partial}{\partial \tilde{x}^{a}}-\frac{i}{2} \tilde{\xi}_{a} \frac{\partial}{\partial \tilde{c}}\right) \\
\tilde{G}_{5}=-i \frac{\partial}{\partial \tilde{\xi}^{5}}-\frac{1}{2} \tilde{\xi}^{5} \frac{\partial}{\partial \tilde{c}_{5}} \\
\tilde{B}_{\alpha a}=-i \tilde{x}_{\alpha} \frac{\partial}{\partial \tilde{x}^{a}}
\end{gathered}
$$

Implying the following variations for the coordinates (omitting the tilde)

$$
\begin{array}{ll}
i \epsilon^{\alpha} G_{\alpha}: \delta \xi^{\alpha}=\epsilon^{\alpha}, & \delta x^{\alpha}=0, \quad \delta c=-\frac{i}{2} \epsilon^{\alpha} \xi_{\alpha} \\
i \epsilon^{a} G_{a}: \delta \xi^{a}=\epsilon^{a}, & \delta x^{a}=i \epsilon^{a} \xi^{5}, \quad \delta c=-\frac{i}{2} \epsilon^{a} \xi_{a}
\end{array}
$$

$$
\begin{gathered}
i \epsilon^{5} G_{5}: \delta \xi^{5}=\epsilon^{5}, \quad \delta c_{5}=-\frac{i}{2} \epsilon^{5} \xi^{5} \\
i v^{\alpha a} B_{\alpha a}: \delta x^{a}=v^{\alpha a} x_{\alpha}
\end{gathered}
$$


(2) $r-t=1$, we get

$$
\begin{gathered}
\tilde{G}_{\alpha}=-i \frac{\partial}{\partial \tilde{\xi}^{\alpha}} \\
\tilde{G}_{a}=-i\left(\frac{\partial}{\partial \tilde{\xi}^{a}}+i \tilde{\xi}^{5} \frac{\partial}{\partial \tilde{x}^{a}}-\frac{i}{2} \tilde{\xi}_{a} \frac{\partial}{\partial \tilde{c}}\right) \\
\tilde{G}_{5}=-i \frac{\partial}{\partial \tilde{\xi}^{5}}-\frac{1}{2} \tilde{\xi}^{5} \frac{\partial}{\partial \tilde{c}_{5}} \\
\tilde{B}_{\alpha a}=-i \tilde{x}_{\alpha} \frac{\partial}{\partial \tilde{x}^{a}}-i \tilde{\xi}_{a} \frac{\partial}{\partial \tilde{\xi}^{\alpha}} .
\end{gathered}
$$

Implying the following variations for the coordinates (omitting the tilde)

$$
\begin{gathered}
i \epsilon^{\alpha} G_{\alpha}: \delta \xi^{\alpha}=\epsilon^{\alpha}, \quad \delta x^{\alpha}=0, \quad \delta c=0 \\
i \epsilon^{a} G_{a}: \delta \xi^{a}=\epsilon^{a}, \quad \delta x^{a}=i \epsilon^{a} \xi^{5}, \quad \delta c=-\frac{i}{2} \epsilon^{a} \xi_{a} \\
i \epsilon^{5} G_{5}: \delta \xi^{5}=\epsilon^{5}, \quad \delta c_{5}=-\frac{i}{2} \epsilon^{5} \xi^{5} \\
i v^{\alpha a} B_{\alpha a}: \delta x^{a}=v^{\alpha a} x_{\alpha}, \quad \delta \xi^{\alpha}=v^{\alpha a} \xi_{a} .
\end{gathered}
$$

In the second case, the boosts depend also on the Grassmann variables $\xi^{\alpha}$ and $\xi^{a}$. Furthermore the VSUSY algebra decomposes in the direct product of a VSUSY in the Euclidean subspace times a Grassmann algebra in the Minkowski part.

\section{THE VSUSY CARROLL PARTICLE}

We start with a Lagrangian invariant under the VSUSY algebra on the total space-time in $D+1$ dimensions [24]

$$
\begin{aligned}
L= & -M \sqrt{-\left(\dot{x}^{\mu}-i \xi^{\mu} \dot{\xi}^{5}\right)^{2}}-\beta\left(\dot{c}+\frac{i}{2} \xi_{\mu} \dot{\xi}^{\mu}\right) \\
& -\gamma\left(\dot{c}_{5}+\frac{i}{2} \xi^{5} \dot{\xi}^{5}\right)
\end{aligned}
$$

This time we will consider a 1-contraction of Carroll type in the case $r-t=0$. The Carroll invariant Lagrangian is obtained restricting (83) to the one-dimensional space spanned by $x^{0}$ and adding its variation under a boost times a lagrange multiplier. Since in this case the boost is operating only on $x^{0}, \delta x^{0}=-\vec{v} \cdot \vec{x}$ [see Eq. (50)], we get

$$
\begin{aligned}
L= & -M \sqrt{\left(\dot{x}^{0}-i \xi^{0} \dot{\xi}^{5}\right)^{2}}-\beta\left(\dot{c}+\frac{i}{2} \xi_{0} \dot{\xi}^{0}\right) \\
& -\gamma\left(\dot{c}_{5}+\frac{i}{2} \xi^{5} \dot{\xi}^{5}\right)+\vec{\lambda} \cdot \dot{\vec{x}}
\end{aligned}
$$

This Lagrangian is in fact VSUSY invariant under the following rigid transformations (see [24])

$$
\begin{gathered}
\delta x^{0}=i \epsilon \xi^{5}, \quad \delta \xi^{0}=\epsilon, \quad \delta \xi^{5}=\epsilon^{5} \\
\delta c=-\frac{i}{2} \epsilon \xi^{0}, \quad \delta c_{5}=-\frac{i}{2} \epsilon^{5} \xi^{5} .
\end{gathered}
$$

The generators of these transformations satisfy the algebra $\left[G_{0}, G_{0}\right]_{+}=-Z, \quad\left[G_{5}, G_{5}\right]_{+}=Z_{5}, \quad\left[G_{0}, G_{5}\right]_{+}=-P_{0}$

where $Z$ and $Z_{5}$ are two central charges. The variation of (84) under Carroll boosts is given by (remember that $\vec{x}$ does not transform under a Carroll boost)

$$
\delta L=p_{0} \delta \dot{x}^{0}+\delta \vec{\lambda} \cdot \dot{\vec{x}}=-p_{0} \vec{v} \cdot \dot{\vec{x}}++\delta \vec{\lambda} \cdot \dot{\vec{x}}
$$

where $p_{0}$ is the canonical momentum associated to $\dot{x}^{0}$

$$
p_{0}=\frac{\partial L}{\partial \dot{x}^{0}}=-M \frac{\left(\dot{x}^{0}-i \xi^{0} \dot{\xi}^{5}\right)}{\sqrt{\left(\dot{x}^{0}-i \xi^{0} \dot{\xi}^{5}\right)^{2}}}
$$

The Lagrangian is Carroll invariant assuming

$$
\delta \vec{\lambda}=p_{0} \vec{v}
$$

The other canonical momenta are

$$
\begin{gathered}
\pi_{0}=\frac{\partial L}{\partial \dot{\xi}^{0}}=\frac{i \beta}{2} \xi_{0} \\
\pi^{5}=\frac{\partial L}{\partial \dot{\xi}^{5}}=\frac{i \gamma}{2} \xi^{5}+i p_{0} \xi^{0} .
\end{gathered}
$$

From these equations the following constraints follow

$$
\begin{gathered}
\phi=p_{0}^{2}-M^{2} \\
\chi=\pi^{5}-\frac{i \gamma}{2} \xi^{5}-i p_{0} \xi^{0} \\
\chi_{0}=\pi_{0}-\frac{i \beta}{2} \xi_{0} .
\end{gathered}
$$

We recall that the Poisson brackets for Grassmann variables are defined by 


$$
\left\{\pi_{0}, \xi^{0}\right\}=-1 .
$$

The constraint (95) is second class. We then define Dirac brackets in the usual way and, in particular, we find

$$
\left\{\xi_{0}, \xi_{0}\right\}^{*}=-\frac{i}{\beta}
$$

We are now able to evaluate the Dirac brackets of the odd constraint $\chi$ with itself. We find

$$
\{\chi, \chi\}^{*}=\frac{i}{\beta}\left(p_{0}^{2}+\beta \gamma\right) .
$$

Therefore, if we choose

$$
\gamma=-\beta=M
$$

the constraints $\phi$ and $\chi$ are first class and we expect, as in the relativistic case, a local kappa symmetry of the Lagrangian.

This model represents the spinning generalization of the Carroll particle model studied in [6]. Although the two cases $t-r=0$ and $t-r=1$ correspond to two different algebras, it is easy to check that the Lagrangian (84) is invariant under also under the algebra corresponding to $r-t=1$.

\section{A. $\kappa$-invariance}

As we have seen, the model has two local symmetries, generated by the constraints $\phi$ and $\chi$. The local symmetry generated by the odd constraint is a " $k$-symmetry." The transformations generated by $\chi$ are given by

$$
\delta x^{0}=i \kappa \xi^{0}, \quad \delta \xi^{0}=\kappa \frac{p^{0}}{M}, \quad \delta \xi^{5}=+\kappa
$$

from which

$$
\begin{aligned}
\delta L= & i \kappa\left(p_{0} \dot{\xi}^{0}+M \dot{\xi}^{5}\right)-\frac{i}{2} \kappa p_{0} \dot{\xi}^{0}-\frac{i}{2} \xi_{0} \frac{d}{d \tau}\left(\kappa p_{0}\right)-\frac{i}{2} M \kappa \dot{\xi}^{5} \\
& -\frac{i}{c} M \xi^{5} \dot{k} \\
= & \frac{i}{2} \kappa p_{0} \dot{\xi}^{0}-\frac{i}{2} \xi^{0} \frac{d}{d \tau}\left(\kappa p_{0}\right)-\frac{i}{2} M \xi^{5} \dot{\kappa}+\frac{i}{2} M \kappa \dot{\xi}^{5} \\
= & \frac{i}{2} \frac{d}{d \tau}\left[\kappa\left(p_{0} \xi^{0}+M \xi^{5}\right)\right] .
\end{aligned}
$$

Therefore the Lagrangian (84) is quasi-invariant under this $\kappa$-transformation.

\section{B. Quantization}

To perform the canonical quantization we require the following conditions for the operators corresponding to the Grassmann variables [25]

$$
\begin{aligned}
& {\left[\hat{\pi}^{5}, \hat{\pi}^{5}\right]_{+}=\left[\hat{\xi}^{5}, \hat{\xi}^{5}\right]_{+}=\left[\hat{\xi}^{5}, \hat{\xi}^{0}\right]_{+}=\left[\hat{\xi}^{0}, \hat{\pi}^{5}\right]_{+}=0,} \\
& {\left[\hat{\pi}^{5}, \hat{\xi}^{5}\right]_{+}=-i, \quad\left[\hat{\xi}_{0}, \hat{\xi}_{0}\right]_{+}=-\frac{1}{M},}
\end{aligned}
$$

we give the following definitions

$\hat{\pi}^{5}=i \sqrt{\frac{M}{2}} P_{1}, \quad \hat{\xi}^{5}=\sqrt{\frac{2}{M}} P_{2}, \quad \hat{\xi}_{0}=\frac{i}{\sqrt{2 M}} \gamma_{0} \gamma_{5}$

where we define the matrix [26]

$$
\gamma_{5}=(-i)^{m+1} \gamma_{0} \gamma_{1} \cdots \gamma_{D}
$$

where $m=(D+1) / 2$, The matrx $\gamma_{5}$ is Hermitian and satisfies

$$
\gamma_{5}^{2}=1
$$

in any space-time of even dimension. The matrices $P_{1}$ and $P_{2}$ are nilpotent and defined by

$$
P_{1}=\frac{\gamma_{0}-\gamma_{5}}{2}, \quad P_{2}=\frac{\gamma_{0}+\gamma_{5}}{2}
$$

such that

$$
P_{1}^{2}=P_{2}^{2}=0, \quad\left[P_{1}, P_{2}\right]_{+}=-1 .
$$

After substitution in the constraint $\chi$ we find the condition on the states

$$
\left(-i \gamma_{0} p^{0} \gamma_{5}-M \gamma_{5}\right) \Psi\left(p^{0}\right)=0 .
$$

Multiplying by $\gamma_{5}$

$$
\left(i \gamma_{0} p^{0}-M\right) \Psi\left(p^{0}\right)=0 .
$$

This shows that the model describes at the same time particles and antiparticles corresponding to the two eigenvalues \pm 1 of $i \gamma_{0}$.

Since there are only 3 operators, the lowest dimensional representation is in terms of $2 \times 2$ matrices. For instance, we can take the following representation which satisfied the previous quantization conditions

$$
\begin{gathered}
\hat{\pi}^{5}=i \sqrt{\frac{M}{2} \frac{\left(i \sigma_{3}-\sigma_{2}\right)}{2}}, \quad \hat{\xi}^{5}=\sqrt{\frac{2}{M}} \frac{\left(i \sigma_{3}+\sigma_{2}\right)}{2}, \\
\tilde{\xi}_{0}=\frac{i}{\sqrt{2 M}} \sigma_{1}
\end{gathered}
$$

where the $\sigma_{i}$ 's are the Pauli matrices. Then the odd constrint $\chi$ becomes 


$$
\left(-i M \sigma_{2}+p^{0} \sigma_{1}\right) \Psi\left(p^{0}\right)=0 .
$$

Multiplying by $\sigma_{1}$

$$
\left(p^{0}+M \sigma_{3}\right) \Psi\left(p^{0}\right)=0 .
$$

Note that we have an ultralocal Dirac equation.

\section{THE VSUSY GALILEI PARTICLE}

We start with a Lagrangian (83) invariant under the VSUSY algebra on the total space-time in $D+1$ dimensions $[11,24]$, in its Euclidean version

$L=M \sqrt{\left(\dot{x}^{\mu}-i \xi^{\mu} \dot{\xi}^{5}\right)^{2}}-\beta\left(\dot{c}+\frac{i}{2} \xi_{\mu} \dot{\xi}^{\mu}\right)-\gamma\left(\dot{c}_{5}+\frac{i}{2} \xi^{5} \dot{\xi}^{5}\right)$

Let us consider a $D$-contraction of the Galilei type for $r-t=0$. According to our philosophy, the Galilei invariant Lagrangian is obtained by using the previous Lagrangian in the euclidean subspace in $D$ dimensions, and then adding its variation under a boost times a lagrangian multiplier. Since in this case the boost is operating only on the space-coordinates, $\delta \vec{x}=\vec{v} x^{0}$ [see Eq. (74)], we get

$$
\begin{aligned}
L= & M \sqrt{\left(\dot{\vec{x}}-i \vec{\xi} \dot{\xi}^{5}\right)^{2}}-\beta\left(\dot{c}+\frac{i}{2} \vec{\xi} \cdot \dot{\vec{\xi}}\right)-\gamma\left(\dot{c}_{5}+\frac{i}{2} \xi^{5} \dot{\xi}^{5}\right) \\
& +\lambda \dot{x}^{0} .
\end{aligned}
$$

This Lagrangian is in fact VSUSY invariant under the following rigid transformations (see [24])

$$
\begin{gathered}
\delta \vec{x}=i \vec{\epsilon} \xi^{5}, \quad \delta \vec{\xi}=\vec{\epsilon}, \quad \delta \xi^{5}=\epsilon^{5} \\
\delta c=-\frac{i}{2} \vec{\epsilon} \cdot \vec{\xi}, \quad \delta c_{5}=-\frac{i}{2} \epsilon^{5} \xi^{5}
\end{gathered}
$$

The generators of these transformations satisfy the algebra

$$
\begin{gathered}
{\left[G_{i}, G_{j}\right]_{+}=Z \delta_{i j}, \quad\left[G_{5}, G_{5}\right]_{+}=Z_{5},} \\
{\left[G_{i}, G_{5}\right]_{+}=-P_{i}}
\end{gathered}
$$

where $Z$ and $Z_{5}$ are two central charges. The variation of (114) under Galilei bosts is given by (remember thgat $x^{0}$ does not transform under a Galilei boost)

$$
\delta L=\vec{p} \cdot \delta \dot{\vec{x}}+\delta \lambda \dot{x}^{0}=\vec{p} \cdot \vec{v} \dot{x}^{0}++\delta \lambda \dot{x}^{0}
$$

where $\vec{p}$ is the canonical momentum

$$
\vec{p}=M \frac{\left(\dot{\vec{x}}-i \vec{\xi} \dot{\xi}^{5}\right)}{\sqrt{\left(\dot{\vec{x}}-i \vec{\xi}^{5}\right)^{2}}}
$$

Therefore the model is Galilei invariant if

$$
\delta \vec{\lambda}=-\vec{p} \cdot \vec{v}
$$

The other odd canonical momenta are

$$
\begin{gathered}
\vec{\pi}=\frac{i \beta}{2} \vec{\xi} \\
\pi^{5}=\frac{i \gamma}{2} \xi^{5}+i \vec{p} \cdot \vec{\xi}
\end{gathered}
$$

From these equations the following constraints follow

$$
\begin{gathered}
\phi=\vec{p}^{2}-M^{2} \\
\chi=\pi^{5}-\frac{i \gamma}{2} \xi^{5}-i \vec{p} \cdot \vec{\xi} \\
\vec{\chi}=\vec{\pi}-\frac{i \beta}{2} \vec{\xi} .
\end{gathered}
$$

The last constraint is second class. We then define Dirac brackets in the usual way and, in particular, we find

$$
\left\{\xi^{i}, \xi^{j}\right\}^{*}=\frac{i}{\beta} \delta^{i j}
$$

We are now able to evaluate the Dirac brackets of the odd constraint $\chi$ with itself. We find

$$
\{\chi, \chi\}^{*}=-\frac{i}{\beta}\left(\vec{p}^{2}-\beta \gamma\right)
$$

Therefore, if we choose

$$
\beta \gamma=M^{2}
$$

the constraints $\phi$ and $\chi$ are first class and we expect, as in the relativistic case, a local kappa symmetry of the Lagrangian.

This model represents the spinning pseudoclassical generalization of the Galileian massless model studied in $[3,4,10]$. The model can be also obtained as the nonrelativistic limit of a tachyonic spinning particle, see the Appendix.

Although the two cases $r-t=0$ and $r-t=1$ correspond to two different algebras, it is easy to check that the Lagrangian (114) is invariant also under the algebra corresponding to $r-t=1$. 


\section{A. $\kappa$-invariance}

As we have seen, the model has two local symmetries, generated by the constraints $\phi$ and $\chi$, if the condition $\beta \gamma=$ $M^{2}$ holds. We will satisfy this condition by the choice $\beta=\gamma=M$. The local symmetry generated by the odd constraint is a " $\kappa$-symmetry." The reasons for this name are twofold. First of all it is a gauge odd-symmetry as the usual $\kappa$-symmetry in SUSY. The second one is that, due to this gauge symmetry, in VSUSY the model has BPS configurations preserving $1 / 5$ of the vector supersymmetry [24].

The transformations generated by $\chi$ are given by

$$
\delta \vec{x}=i \kappa \vec{\xi}, \quad \delta \vec{\xi}=\kappa \frac{\vec{p}}{M}, \quad \delta \xi^{5}=-\kappa
$$

we find

$$
\begin{aligned}
\delta L= & i \kappa\left(\vec{p} \cdot \dot{\vec{\xi}}-M \dot{\xi}^{5}\right)-\frac{i}{2} \kappa \vec{p} \cdot \dot{\vec{\xi}}-\frac{i}{2} \vec{\xi} \cdot \frac{d}{d \tau}(\kappa \vec{p})+\frac{i}{2} M \kappa \dot{\xi}^{5} \\
& +\frac{i}{c} M \xi^{5} \dot{k} \\
= & \frac{i}{2} \kappa \vec{p} \cdot \dot{\vec{\xi}}+\frac{i}{2} \frac{d}{d \tau}(\kappa \vec{p}) \cdot \vec{\xi}+\frac{i}{2} M \xi^{5} \dot{\kappa}+\frac{i}{2} M \kappa \dot{\xi}^{5} \kappa \\
= & \frac{i}{2} \frac{d}{d \tau}\left[\kappa\left(\vec{p} \cdot \vec{\xi}-M \xi^{5}\right)\right]
\end{aligned}
$$

where we have used $\beta \gamma=M^{2}$. Therefore the Lagrangian (114) is quasi-invariant under this $\kappa$-transformation.

\section{B. Quantization}

To perform the canonical quantization we require the following conditions for the operators corresponding to the Grassmann variables [25]

$$
\begin{aligned}
& {\left[\hat{\pi}^{5}, \hat{\pi}^{5}\right]_{+}=\left[\hat{\xi}^{5}, \hat{\xi}^{5}\right]_{+}=\left[\hat{\xi}^{5}, \hat{\xi}_{i}\right]_{+}=\left[\hat{\xi}_{i}, \hat{\pi}^{5}\right]_{+}=0,} \\
& {\left[\hat{\pi}^{5}, \hat{\xi}^{5}\right]_{+}=-i, \quad\left[\hat{\xi}_{i}, \hat{\xi}_{j}\right]_{+}=-\frac{1}{M} \delta_{i j}}
\end{aligned}
$$

In this context we will suppose to be in a space-time of even dimensions $D+1=2 m$. The $\gamma$-matrices satisfy

$$
\left[\gamma_{\mu}, \gamma_{\nu}\right]_{+}=2 g_{\mu \nu}
$$

Then, we see that the anticommutators in Eq. (131) are satisfied by

$$
\hat{\pi}^{5}=i \sqrt{\frac{M}{2}} P_{1}, \quad \hat{\xi}^{5}=\sqrt{\frac{2}{M}} P_{2}, \quad \hat{\xi}_{i}=\frac{i}{\sqrt{2 M}} \gamma_{i}
$$

where $P_{1}$ and $P_{2}$ are defined in (106). Substituting these expressions inside the constraint (124) we find that the physical states must satisfy the equation

$$
\left(\vec{p} \cdot \vec{\gamma}-i M \gamma_{5}\right) \Psi(\vec{p})=0
$$

or, multiplying by $i \gamma_{5}$

$$
\left(i \vec{p} \cdot \vec{\gamma} \gamma_{5}-M\right) \Psi(\vec{p})=0
$$

Then, define

$$
\Gamma_{i}=i \gamma_{i} \gamma_{5}
$$

which satisfy

$$
\left[\Gamma_{i}, \Gamma_{j}\right]_{+}=2 \delta_{i j}
$$

Therefore the $\Gamma_{i}$ 's give a representation of the Clifford algebra in a space of $D$ dimensions equivalent to the one spanned by the $\gamma_{i}$ 's. In this way we get

$$
(\vec{p} \cdot \vec{\Gamma}-M) \Psi(\vec{p})=0
$$

which is the Dirac equation in a Euclidean space of odd dimensions $D$. After this equation is satisfied, the physical states satisfy automatically the condition arising from the even constraint $\phi$

$$
\left(\vec{p}^{2}-M^{2}\right) \Psi(\vec{p})=0 .
$$

Notice also that the lower dimensional representation of the Clifford algebra in $D$ odd dimensions is through $2^{(D-1) / 2} \times$ $2^{(D-1) / 2}$ matrices. For instance in 3-dimensions the lowest dimensional representation is by the $2 \times 2$ Pauli matrices.

\section{CONCLUSIONS}

In this paper we have introduced two models of pseudoclassical [25] spinning particles respectively invariant under the Carroll and the Galilei groups with zero central charge. The construction of these models has been done through a generalization of the method introduced in Ref. [1]. The method consists in starting from a space-time in $D+1$ dimensions and partitioning it in two parts, the first Minkowskian and the second Euclidean. Then, in the Carroll case an invariant model can be obtained by introducing a Minkowski invariant action in the first part of the space-time and in the second part a system of Lagrange multipliers transforming in an appropriate way under the Euclidean group such to confine the system to a region of the space-time. An analogous procedure can be followed in the Galilei case.

This procedure allows us to construct a class of Carroll and Galilei invariant models, once given a relativistic action in configuration space without performing a Carroll or nonrelativistic limit. In the two particular cases discussed here, we have started from an action in configuration space invariant under the VSUSY algebra [11,24]. The two models turn out to be the pseudoclassical spinning version 
of the Carroll particle described in Ref. [6] and of the Galilei particle of Refs. $[3,4,10]$. We have also checked that our results can be reproduced starting from the VSUSY invariant model and performing the standard Carroll and Galilei limiting procedure. The limiting procedure is consistent with the generalized contractions of the VSUSY algebra consistent with the contractions of the Poincaré subalgebra discussed in [1].

We have performed a detailed analysis of these models, showing that they preserve the same features of the VSUSY invariant model. Namely, the presence of two constraints, one even corresponding to the mass-shell condition and an odd one, leading to the relevant analogous of the Dirac equation, after quantization. We have also shown that with a convenient choice of the parameters appearing in the Lagrangians, the models both possess a $\kappa$-symmetry.

\section{ACKNOWLEDGMENTS}

J. G. has been supported in part by Programa Nacional de Física de Partículas (FPA) 2013-46570-C2-1-P and Consolider Centro Nacional de Física de Particulas, Astropartículas y Nuclear (CPAN) and by the Spanish government (MINECO/FEDER) under Project No. MDM2014-0369 of Institut de Ciencies del Cosmos Universitat de Barcelona (Unidad de Excelencia Maria de Maeztu). J. G. has also been supported by CONICYT under Grant No. PAI801620047 as a visiting professor of the Universidad Austral de Chile.

\section{APPENDIX: NONRELATICISTIC LIMITS}

In this Appendix we will show that for the two models presented here, our strategy is equivalent to take the usual Galilei and Carroll limit from the relativistic phase space Lagrangian.

The canonical Lagrangian of a relativistic spinning tachyon is given by

$$
L^{C}=p_{\mu}\left(\dot{x}^{\mu}-i \xi^{\mu} \dot{\xi}^{5}\right)-\beta \frac{i}{2} \xi_{\mu} \dot{\xi}^{\mu}-\gamma \frac{i}{2} \xi^{5} \dot{\xi}^{5}-\frac{e}{2}\left(p^{2}-m^{2}\right)
$$

or in symplectic form

$$
\begin{aligned}
L^{S}= & p_{\mu} \dot{x}^{\mu}-\beta \frac{i}{2} \xi_{\mu} \dot{\xi}^{\mu}-\pi_{5} \dot{\xi}^{5}-\frac{e}{2}\left(p^{2}-m^{2}\right) \\
& -\rho\left(\pi_{5}-\gamma \frac{i}{2} \xi^{5}-i p_{\mu} \xi^{\mu}\right)
\end{aligned}
$$

where

$$
\chi_{5}=\pi_{5}-\gamma \frac{i}{2} \xi^{5}-i p_{\mu} \xi^{\mu}
$$

The Poisson-Dirac brackets are

$$
\begin{gathered}
\left\{p_{\mu}, x^{\nu}\right\}^{*}=-\delta_{\mu}{ }^{\nu}, \quad\left\{\xi^{\mu}, \xi^{\nu}\right\}^{*}=\frac{i}{\beta} \eta^{\mu \nu}, \\
\left\{\pi_{5}, \xi^{5}\right\}^{*}=-1 \\
\left\{\chi_{5}, \chi_{5}\right\}^{*}=i \gamma-\frac{i}{\beta} p_{\mu} p_{\nu} \eta^{\mu \nu}=-\frac{2 i}{\beta} \frac{e}{2}\left(p^{2}-m^{2}\right),
\end{gathered}
$$

where $\beta=\gamma=m . \chi_{5}$ generates the local kappa variation.

\section{A. Nonrelativistic (Galilei) limit}

We define the nonrelativistic limit as

$$
\begin{array}{rlrl}
x^{0} & =\omega t, & & p_{0}=-\frac{E}{\omega}, \\
\xi^{5}=\xi^{5}, & \pi_{5}=\pi_{5}
\end{array}
$$

in the limit $\omega \rightarrow \infty$ we have the action

$$
\begin{aligned}
L^{\mathrm{NR}}= & -E \dot{t}+\vec{p} \dot{\vec{x}}-\beta \frac{i}{2} \xi_{\mu} \dot{\xi}^{\mu}-\pi_{5} \dot{\xi}^{5}-\frac{e}{2}\left(\vec{p}^{2}-m^{2}\right) \\
& -\rho\left(\pi_{5}-\gamma \frac{i}{2} \xi^{5}-i \vec{p} \vec{\xi}\right),
\end{aligned}
$$

which in the reduced space is equivalent to the Hamiltonian form of (114). The structure of Poisson brackets is maintained for the nonrelativisitic variables. Also the first class character of constraints is maintained.

\section{B. Carroll limit}

The symplectic action of a relativistic particle is

$$
\begin{aligned}
L^{S}= & p_{\mu} \dot{x}^{\mu}-\beta \frac{i}{2} \xi_{\mu} \dot{\xi}^{\mu}-\pi_{5} \dot{\xi}^{5}-\frac{e}{2}\left(p^{2}+m^{2}\right) \\
& -\rho\left(\pi_{5}-\gamma \frac{i}{2} \xi^{5}-i p_{\mu} \xi^{\mu}\right),
\end{aligned}
$$

where $\beta=-m, \gamma=m$.

We define the Carroll limit as

$$
\begin{array}{rlrl}
x^{0} & =\frac{1}{\omega} t, & p_{0}=-\omega E, & \xi^{\mu}=\frac{1}{\sqrt{\omega}} \xi^{\mu}, \\
\xi^{5}=\frac{1}{\sqrt{\omega}} \xi^{5}, & \pi_{5}=\omega \pi_{5}, & m=\omega M
\end{array}
$$

in the limit $\omega \rightarrow \infty$ we have the action

$$
\begin{aligned}
L^{\text {Carroll }}= & -E \dot{t}+\vec{p} \dot{\vec{x}}-\beta \frac{i}{2} \xi_{\mu} \dot{\xi}^{\mu}-\pi_{5} \dot{\xi}^{5} \\
& -\frac{\tilde{e}}{2}\left(E^{2}-M^{2}\right)-\tilde{\rho}\left(\pi_{5}-\gamma \frac{i}{2} \xi^{5}+i E \xi^{0}\right),
\end{aligned}
$$

where $\tilde{e}=-\omega^{2} e, \tilde{\rho}=\sqrt{\omega} \rho$, which in the reduced space is equivalent to the Hamiltonian form of (114). The PoissonDirac brackets and the character of the constraints is maintained. 
[1] A. Barducci, R. Casalbuoni, and J. Gomis, Confined dynamical systems with Carroll or Galilei symmetries, Phys. Rev. D 98, 085018 (2018).

[2] J. M. Lévy-Leblond, Une nouvelle limite non-relativiste du group de Poincaré, Ann. Inst. Henri Poincaré 3, 1 (1965); V. D. Sen Gupta, On an analogue of the Galileo group, Il Nuovo Cimento 54, 512 (1966).

[3] J.-M. Souriau, Structure des systèmes dynamiques (Dunod, 1970); Structure of Dynamical Systems: A Symplectic View of Physics, translated by C. H. Cushman-de Vries (R. H. Cushman and G. M. Tuynman Translation Editors) (Birkhäuser, 1997).

[4] C. Duval and P. A. Horvathy, Non-relativistic conformal symmetries and Newton-Cartan structures, J. Phys. A 42, 465206 (2009).

[5] C. Duval, G. W. Gibbons, P. A. Horvathy, and P. M. Zhang, Carroll versus Newton and Galilei: Two dual non-Einsteinian concepts of time, Classical Quantum Gravity 31, 085016 (2014).

[6] E. Bergshoeff, J. Gomis, and G. Longhi, Dynamics of Carroll particles, Classical Quantum Gravity 31, 205009 (2014).

[7] B. Cardona, J. Gomis, and J. M. Pons, Dynamics of Carroll strings, J. High Energy Phys. 07 (2016) 050.

[8] C. Batlle, J. Gomis, and D. Not, Extended Galilean symmetries of non-relativistic strings, J. High Energy Phys. 02 (2017) 049.

[9] J. Gomis and P. K. Townsend, The Galilean superstring, J. High Energy Phys. 02 (2017) 105.

[10] C. Batlle, J. Gomis, L. Mezincescu, and P. K. Townsend, Tachyons in the Galilean limit, J. High Energy Phys. 04 (2017) 120.

[11] A. Barducci, R. Casalbuoni, and L. Lusanna, Supersymmetries and the Pseudoclassical relativistic electron, Nuovo Cimento A 35, 377 (1976).

[12] L. Brink, S. Deser, B. Zumino, P. Di Vecchia, and P. S. Howe, Local supersymmetry for spinning particles, Phys. Lett. 64B, 435 (1976);
[13] L. Brink, P. Di Vecchia, and P. S. Howe, A Lagrangian formulation of the classical and quantum dynamics of spinning particles, Nucl. Phys. B118, 76 (1977).

[14] F. A. Berezin and M. S. Marinov, Particle spin dynamics as the Grassmann variant of classical mechanics, Ann. Phys. (N.Y.) 104, 336 (1977).

[15] R. Casalbuoni, J. Gomis, and K. Kamimura, SuperParticle realization of twisted $N=2$ SUSY algebra, J. High Energy Phys. 01 (2011) 098.

[16] K. Costello and S. Li, Twisted supergravity and its quantization, arXiv:1606.00365.

[17] R. Casalbuoni, F. Elmetti, J. Gomis, K. Kamimura, L. Tamassia, and A. Van Proeyen, Vector supersymmetry: Casimir operators and contraction from OSp(3,2-2), J. High Energy Phys. 01 (2009) 035.

[18] J. Gomis and H. Ooguri, Nonrelativistic closed string theory, J. Math. Phys. (N.Y.) 42, 3127 (2001).

[19] U. H. Danielsson, A. Guijosa, and M. Kruczenski, IIA/B, wound and wrapped, J. High Energy Phys. 10 (2000) 020.

[20] J. Brugues, T. Curtright, J. Gomis, and L. Mezincescu, Nonrelativistic strings and branes as non-linear realizations of Galilei groups, Phys. Lett. B 594, 227 (2004).

[21] J. Gomis, J. Gomis, and K. Kamimura, Non-relativistic superstrings: A new soluble sector of $\mathrm{AdS}_{5} \times S^{5}$, J. High Energy Phys. 12 (2005) 024.

[22] J. Brugues, J. Gomis, and K. Kamimura, Newton-Hooke algebras, non-relativistic branes and generalized pp-wave metrics, Phys. Rev. D 73, 085011 (2006).

[23] J.-M. Lévy-Leblond, Galilei Group and Galilean Invariance, in Group Theory and Applications, Loebl ed., vol. II (Academic Press, New York, 1972), p. 222.

[24] R. Casalbuoni, J. Gomis, K. Kamimura, and G. Longhi, Space-time vector supersymmetry and massive spinning particle, J. High Energy Phys. 02 (2008) 094.

[25] R. Casalbuoni, On the quantization of systems with anticommutating variables, Nuovo Cimento A 33, 115 (1976).

[26] D.Z. Freedman and A. Van Proeyen, Supergravity (Cambridge University Press, Cambridge, England, 2012). 